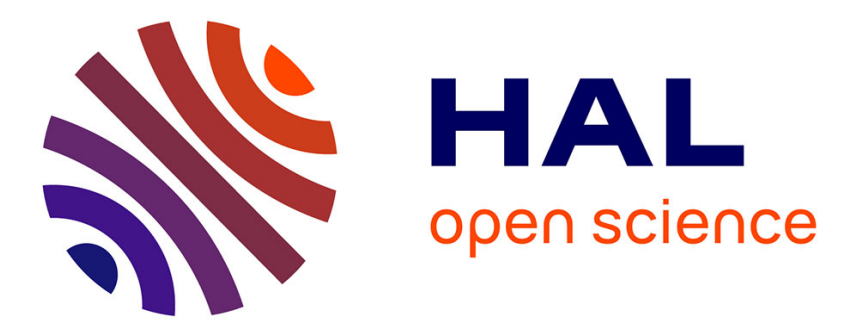

\title{
Electric-field control of magnetic order above room temperature
}

\author{
R.O. Cherifi, V. Ivanovskaya, L.C. Phillips, A. Zobelli, I.C. Infante, E. \\ Jacquet, V. Garcia, S. Fusil, P.R. Briddon, N. Guiblin, et al.
}

\section{- To cite this version:}

R.O. Cherifi, V. Ivanovskaya, L.C. Phillips, A. Zobelli, I.C. Infante, et al.. Electric-field control of magnetic order above room temperature. Nature Materials, 2014, pp.nmat3870. 10.1038/NMAT3870 . hal-01053074

\section{HAL Id: hal-01053074 https://hal.science/hal-01053074}

Submitted on 24 Nov 2014

HAL is a multi-disciplinary open access archive for the deposit and dissemination of scientific research documents, whether they are published or not. The documents may come from teaching and research institutions in France or abroad, or from public or private research centers.
L'archive ouverte pluridisciplinaire HAL, est destinée au dépôt et à la diffusion de documents scientifiques de niveau recherche, publiés ou non, émanant des établissements d'enseignement et de recherche français ou étrangers, des laboratoires publics ou privés. 


\title{
Electric-field control of magnetic order above room temperature
}

\author{
R. O. Cherifi ${ }^{1 \dagger}$, V. Ivanovskaya ${ }^{1 \dagger}$, L. C. Phillips ${ }^{1}$, A. Zobelli ${ }^{2}$, I. C. Infante ${ }^{3}$, E. Jacquet ${ }^{1}$, V. Garcia ${ }^{1}$, \\ S. Fusil ${ }^{1,4}$, P. R. Briddon ${ }^{5}$, N. Guiblin ${ }^{3}$, A. Mougin ${ }^{2}$, A. A. Ünal ${ }^{6}$, F. Kronast ${ }^{6}$, S. Valencia ${ }^{6}$, B. Dkhil ${ }^{3}$, \\ A. Barthélémy ${ }^{1}$ and $M$. Bibes ${ }^{1 \star}$
}

Controlling magnetism by means of electric fields is a key issue for the future development of low-power spintronics ${ }^{1}$. Progress has been made in the electrical control of magnetic anisotropy ${ }^{2}$, domain structure ${ }^{3,4}$, spin polarization ${ }^{5,6}$ or critical temperatures ${ }^{7,8}$. However, the ability to turn on and off robust ferromagnetism at room temperature and above has remained elusive. Here we use ferroelectricity in $\mathrm{BaTiO}_{3}$ crystals to tune the sharp metamagnetic transition temperature of epitaxially grown FeRh films and electrically drive a transition between antiferromagnetic and ferromagnetic order with only a few volts, just above room temperature. The detailed analysis of the data in the light of first-principles calculations indicate that the phenomenon is mediated by both strain and field effects from the $\mathrm{BaTiO}_{3}$. Our results correspond to a magnetoelectric coupling larger than previous reports by at least one order of magnitude and open new perspectives for the use of ferroelectrics in magnetic storage and spintronics.

The $\alpha^{\prime}$ phase (CsCl-type) of near equiatomic alloys of $\mathrm{Fe}$ and Rh exhibits a peculiar first-order transition from antiferromagnetic (AFM) order to ferromagnetic (FM) order around $T^{*} \approx 350 \mathrm{~K}$ (ref. 9). In the FM state, the Fe and Rh atoms carry parallel magnetic moments of $\sim 2.2 \mu \mathrm{B}$ and $\sim 0.7 \mu \mathrm{B}$ (ref. 10), respectively, and the magnetic Curie temperature is about $700 \mathrm{~K}$ (ref. 9). Remarkably, the AFM-to-FM metamagnetic transition is accompanied by a $\sim 1 \%$ change in the unit cell volume ${ }^{9}$, indicating a substantial coupling between magnetic and structural properties (as also revealed by the dependence of $T^{*}$ on hydrostatic pressure ${ }^{9,11}$ ). In addition, Hall ${ }^{12}$ and photoemission data ${ }^{13}$ have evidenced strong changes in the electronic structure between both magnetic states, with an unusually low carrier density in the AFM phase ${ }^{12}$. These two features suggest that FeRh is a good candidate for the electrical control of magnetism ${ }^{14,15}$ using ferroelectrics through piezoelectricity (which produces voltage-induced strain) and the field effect (which depletes or accumulates carriers in a material adjacent to the ferroelectric).

Here we report the epitaxial growth of FeRh films on ferroelectric $\mathrm{BaTiO}_{3}$ single crystals and the influence of a voltage applied across $\mathrm{BaTiO}_{3}$ on the magnetic response of the FeRh. We find that electric fields as low as $E=0.4 \mathrm{kV} \mathrm{cm}^{-1}$ increase $T^{*}$ by about $25 \mathrm{~K}$, which is enough to isothermally convert the FeRh from AFM to a largely FM state, with maximum electric-field-induced changes in magnetization of $\sim 550$ e.m.u. $\mathrm{cm}^{-3}$. The dependence of the magnetization on the electric field is relatively symmetric, indicating that this giant magnetoelectric response is mainly driven by voltage-induced strain, with electrostatic effects playing a smaller role. Our first-principles calculations for both mechanisms account for existing literature data and qualitatively explain our experimental results.

FeRh films $22 \mathrm{~nm}$ in thickness were grown at $630^{\circ} \mathrm{C}$ by rfsputtering from a stoichiometric $\mathrm{Fe}_{50} \mathrm{Rh}_{50}$ target onto (001)oriented $\mathrm{BaTiO}_{3}$ single crystals. After growth, the films were annealed in situ at $730^{\circ} \mathrm{C}$ and $P=8.5 \times 10^{-8}$ mbar for $90 \mathrm{~min}$. On the back side of the $\mathrm{BaTiO}_{3}$, we sputtered a 50-nm-thick Au layer. Figure 1a shows reflection high-energy electron diffraction (RHEED) images of the $\mathrm{BaTiO}_{3}$ crystal and of the FeRh film, for the electron beam parallel to the $\mathrm{BaTiO}_{3}$ [100] (left) and [110] (right) directions. The FeRh RHEED images correspond to a bodycentred cubic polytype with a rather flat surface and the epitaxial relationships [100] FeRh \| [110] $\mathrm{BaTiO}_{3}$ and (001)FeRh $\|$ (001)BTO. $\mathrm{X}$-ray diffraction scans confirmed the epitaxy of $\alpha^{\prime}$-FeRh with an excellent $\mathrm{Fe} / \mathrm{Rh}$ order parameter $S=0.96$, calculated from the relative integrated intensity of the (001) and (002) peaks ${ }^{12}$. Minute amounts of $\gamma-\mathrm{Fe}_{2} \mathrm{O}_{3}$ were also detected, probably reflecting a slight oxidation of the surface of the (uncapped) FeRh film. Between 290 and $400 \mathrm{~K}, \mathrm{BaTiO}_{3}$ is tetragonal, with a unit cell that is elongated along the tetragonal axis, which coincides with the ferroelectric polarization direction. (001)-oriented $\mathrm{BaTiO}_{3}$ crystals are split into domains in which the polarization can lie out of the plane (c-type domains) or in the plane (a-type domains). As illustrated in Fig. 1b, the $\mathrm{BaTiO}_{3}$ substrate showed sets of double peaks, indicating the presence of both domain types. At $300 \mathrm{~K}$ the lattice parameters are $c_{\mathrm{BTO}}=4.032 \AA$ and $a_{\mathrm{BTO}}=3.989 \AA$ for $\mathrm{BaTiO}_{3}$ and $c_{\mathrm{FeRh}}=2.995 \AA$ for FeRh, in good agreement with literature values ${ }^{16,17}$. On warming above $300 \mathrm{~K}, c_{\mathrm{FeRh}}$ stays constant near $3.000 \AA$ up to $370 \mathrm{~K}$ and then increases to $3.022 \AA$ at $440 \mathrm{~K}$, Fig. 1c. This signals the transition from the AFM state to the FM state ${ }^{9}$ (see the magnetic hysteresis loops collected by means of the magneto-optical Kerr effect at 300 and $430 \mathrm{~K}$ in Fig. 1e). Cooling down to room temperature, $c_{\mathrm{FeRh}}$ decreases back to its original value, with a thermal hysteresis typical of this first-order metamagnetic transition ${ }^{9}$.

Figure 1d presents the temperature dependence of the magnetization below $320 \mathrm{~K}$ for an $\mathrm{FeRh} / \mathrm{BaTiO}_{3}$ sample. As widely reported, the magnetization is not strictly zero in the nominally AFM phase ${ }^{18}$, but small kinks are noticeable at the structural phase

\footnotetext{
1Unité Mixte de Physique CNRS/Thales, 1 av. Fresnel, 91767 Palaiseau \& Université Paris-Sud, Orsay 91405, France, 2Laboratoire de Physique des Solides, Université Paris-Sud, CNRS UMR 8502, Orsay 91405, France, ${ }^{3}$ Laboratoire SPMS, UMR 8580, Ecole Centrale Paris-CNRS, Grande voie des vignes, Châtenay-Malabry 92290, France, ${ }^{4}$ Université d'Evry-Val d'Essonne, Bd. F. Mitterrand, Evry cedex 91025, France, ${ }^{5}$ School of Electrical and Electronic Engineering, University of Newcastle, Newcastle upon Tyne, NE $17 R U$, UK, ${ }^{6}$ Helmholtz-Zentrum Berlin für Materialen und Energie, Albert-Einstein-Strasse 15, Berlin 12489, Germany. †These authors contributed equally to this work. *email: manuel.bibes@thalesgroup.com
} 
a

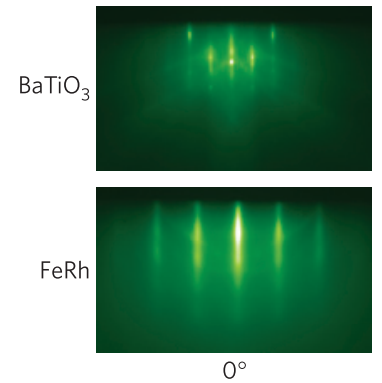

$0^{\circ}$

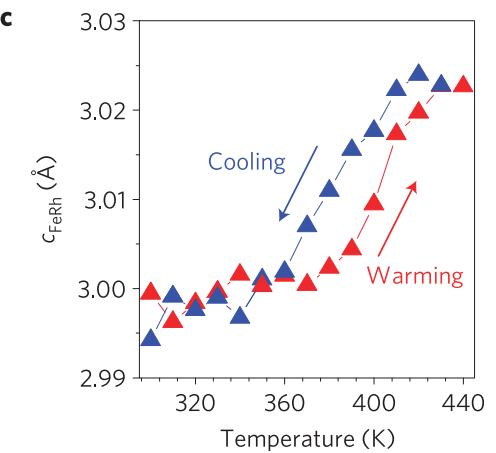

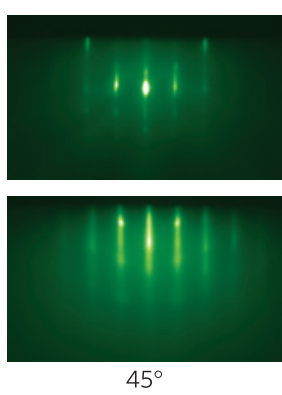

d

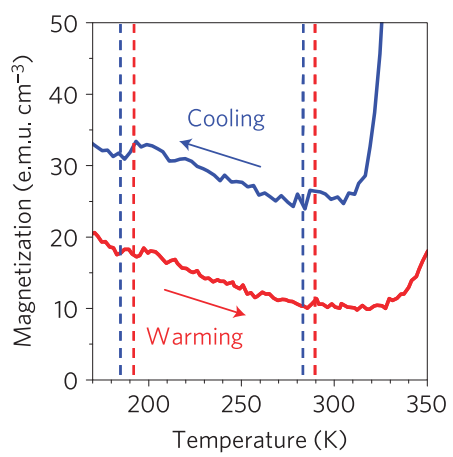

b

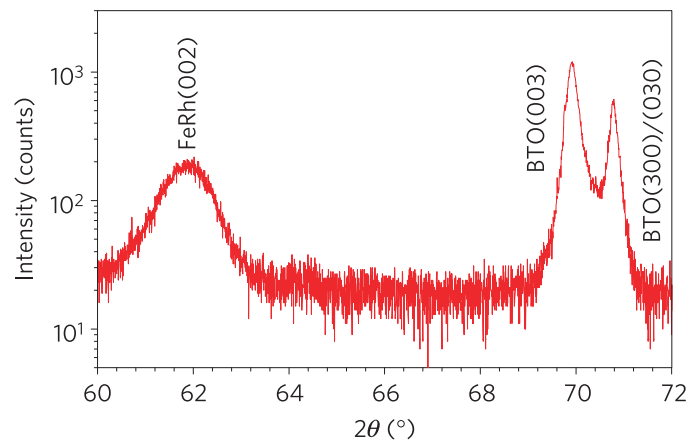

e

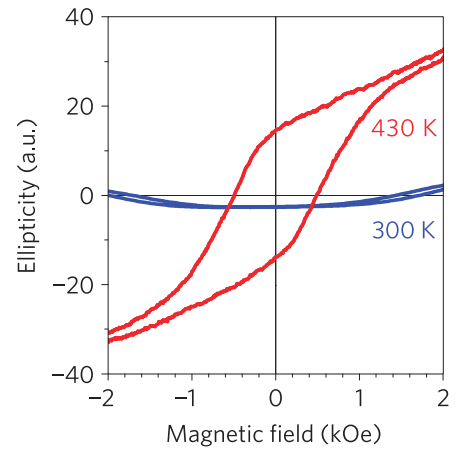

Figure 1 | Structural and magnetic properties of $\mathrm{FeRh}_{\mathbf{B a T i O}} \mathbf{3} \cdot \mathbf{a}, \mathrm{RHEED}$ images of the $\mathrm{BaTiO}_{3}$ substrate before growth and the FeRh after growth and annealing, at two azimuths, $0^{\circ}$ and $45^{\circ}$ from the [100] direction of $\mathrm{BaTiO}_{3} \cdot \mathbf{b}, 2 \theta-\omega$ scan at $300 \mathrm{~K}$ near the FeRh(002) peak at the $\mathrm{BTO}(003)$ and (300)/(030) reflections. c, Temperature dependence of the out-of-plane parameter of FeRh. d, Temperature dependence of the magnetization measured at $20 \mathrm{kOe}$. e, Kerr ellipticity as a function of magnetic field at two different temperatures.

transitions of $\mathrm{BaTiO}_{3}$ - that is, rhombohedral to orthorhombic near $190 \mathrm{~K}$ and orthorhombic to tetragonal near $290 \mathrm{~K}$. On the cooling run, the kinks are shifted down by about $10 \mathrm{~K}$, as expected from the first-order nature of these structural transformations of $\mathrm{BaTiO}_{3}$ (ref. 17).

Figure 2a shows a typical ferroelectric loop for an $\mathrm{FeRh} / \mathrm{BaTiO}_{3}$ sample, measured at $300 \mathrm{~Hz}$ and room temperature. Positive voltage orients the polarization towards the FeRh bottom electrode. A clear hysteretic dependence of the polarization with applied voltage is observed, with a saturation polarization of $12 \mu \mathrm{Ccm}^{-2}$ and coercive voltages of about $20 \mathrm{~V}$. To probe the influence of ferroelectricity on the magnetic response of FeRh, temperaturedependent magnetization $(M)$ measurements were performed with and without an applied voltage in a magnetic field of $20 \mathrm{kOe}$ (which typically shifts $T^{*}$ down by $15-20 \mathrm{~K}$; ref. 19). The top panel in Fig. $2 \mathrm{~b}$ shows an $M(T)$ curve measured in the virgin state of the $\mathrm{BaTiO}_{3}$ (circled 1, Fig. 2a), before the application of any external voltage. As the temperature increases beyond $T^{*}=350 \mathrm{~K}$, the magnetization rises from about 50 e.m.u. $\mathrm{cm}^{-3}$ to nearly 800 e.m.u. $\mathrm{cm}^{-3}$ at $400 \mathrm{~K}$ (the maximum temperature accessible in our superconducting quantum interference device (SQUID) magnetometer), signalling the transition from the AFM to the FM state. On cooling, the magnetization decreases to recover a low value typical of the AFM state, with a thermal hysteresis of about $30 \mathrm{~K}$. The hysteresis is broader than in bulk FeRh (ref. 9), probably as a result of substrate-induced strain. Repeating this experiment with a constant d.c. voltage of $+21 \mathrm{~V}$ applied across the sample (circled 2, Fig. 2a) reveals that the onset of FM order is now shifted up by $25 \mathrm{~K}\left(T^{*}=375 \mathrm{~K}\right)$. At $400 \mathrm{~K}$ the transition is not complete and the magnetization reaches 380 e.m.u. $\mathrm{cm}^{-3}$. On cooling, the magnetization decreases hysteretically and recovers its value in the AFM phase at about $360 \mathrm{~K}$. An $M(T)$ curve taken after turning off the voltage (circled 3, Fig. 2a) seems similar to that of the curve circled 1, albeit with a slightly smaller magnetization value at $400 \mathrm{~K}$.
With a negative voltage now applied ( $-21 \mathrm{~V}$; circled 4, Fig. 2a) $T^{*}$ is again shifted to high temperatures, but less than with a positive voltage. After turning the voltage off (circled 5, Fig. 2a), the initial state is again restored.

The data of Fig. 2b clearly show that the application of a voltage across the $\mathrm{BaTiO}_{3}$ shifts the metamagnetic transition by $\sim 25 \mathrm{~K}$, a temperature interval comparable to the width of the transition $(\sim 50 \mathrm{~K}$ in our films). For temperatures between $\sim 350$ and $400 \mathrm{~K}$, the magnetization is drastically changed by the application of the voltage, with maximum variations reaching $\Delta M=550$ e.m.u. $\mathrm{cm}^{-3}$, Fig. 2c. This corresponds to a gigantic magnetoelectric coupling $\alpha=\mu_{0} \Delta M / E=1.6 \times 10^{-5} \mathrm{sm}^{-1}$, which is five orders of magnitude larger than in single-phase materials $\left(\alpha=3 \times 10^{-10} \mathrm{sm}^{-1}\right.$ in $\mathrm{TbPO}_{4}$; ref. 20) and much higher than previous reports in composite multiferroic heterostructures (for example, $\mathrm{BaTiO}_{3} / \mathrm{La}_{0.67} \mathrm{Sr}_{0.33} \mathrm{MnO}_{3}, \alpha=2.3 \times 10^{-7} \mathrm{sm}^{-1}$; ref. 21; $\mathrm{Pb}\left(\mathrm{Mg}_{1 / 3} \mathrm{Nb}_{2 / 3}\right)_{0.7} \mathrm{Ti}_{0.3} \mathrm{O}_{3} / \mathrm{Co}_{40} \mathrm{Fe}_{40} \mathrm{~B}_{20}, \alpha=2 \times 10^{-6} \mathrm{~s} \mathrm{~m}^{-1}$; ref. 22).

Figure $3 \mathrm{a}$ presents the dependence of the magnetization with voltage at $385 \mathrm{~K}$, after warming from room temperature with a voltage of $+21 \mathrm{~V}$ applied to the sample. On sweeping the voltage from this value towards $-21 \mathrm{~V}$, the magnetization increases from 50 e.m.u. $\mathrm{cm}^{-3}$ to a maximum of 320 e.m.u. $\mathrm{cm}^{-3}$ near $-8 \mathrm{~V}$, and then decreases to 265 e.m.u. $\mathrm{cm}^{-3}$ at $-21 \mathrm{~V}$. Sweeping back to high positive voltage first raises the magnetization to the same maximum value at about $+1 \mathrm{~V}$; as the voltage reaches $+21 \mathrm{~V}$ again $M$ then decreases to 240 e.m.u. $\mathrm{cm}^{-3}$. Returning to $-21 \mathrm{~V}$ produces a hysteretic dependence, with again a local maximum near $-8 \mathrm{~V}$. These data thus confirm the results of Fig. $2 b$ and show that very large magnetization changes can be induced by small electric fields in $\mathrm{FeRh} / \mathrm{BaTiO}_{3}$ heterostructures. The variation is larger for the first sweep $\left(\Delta M=270\right.$ e.m.u. $\left.\mathrm{cm}^{-3}\right)$, presumably owing to the first-order nature of the metamagnetic transition of FeRh. This $\Delta M$ agrees fairly well with the value obtained by subtracting the curves circled 3 and circled 2, Fig. 2c. Past this irreversible 
a
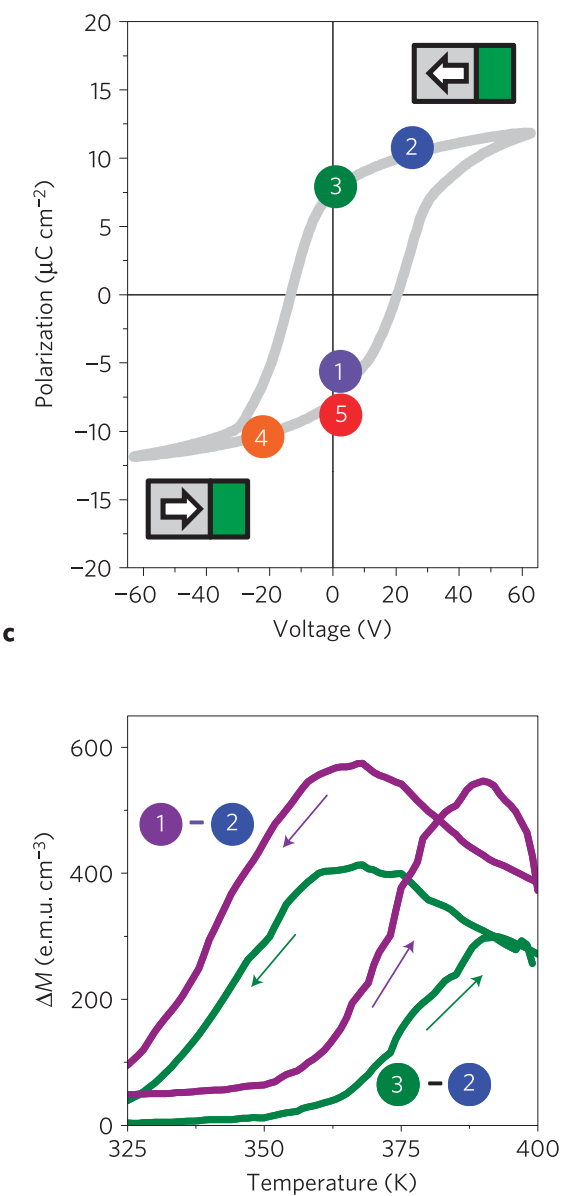

b

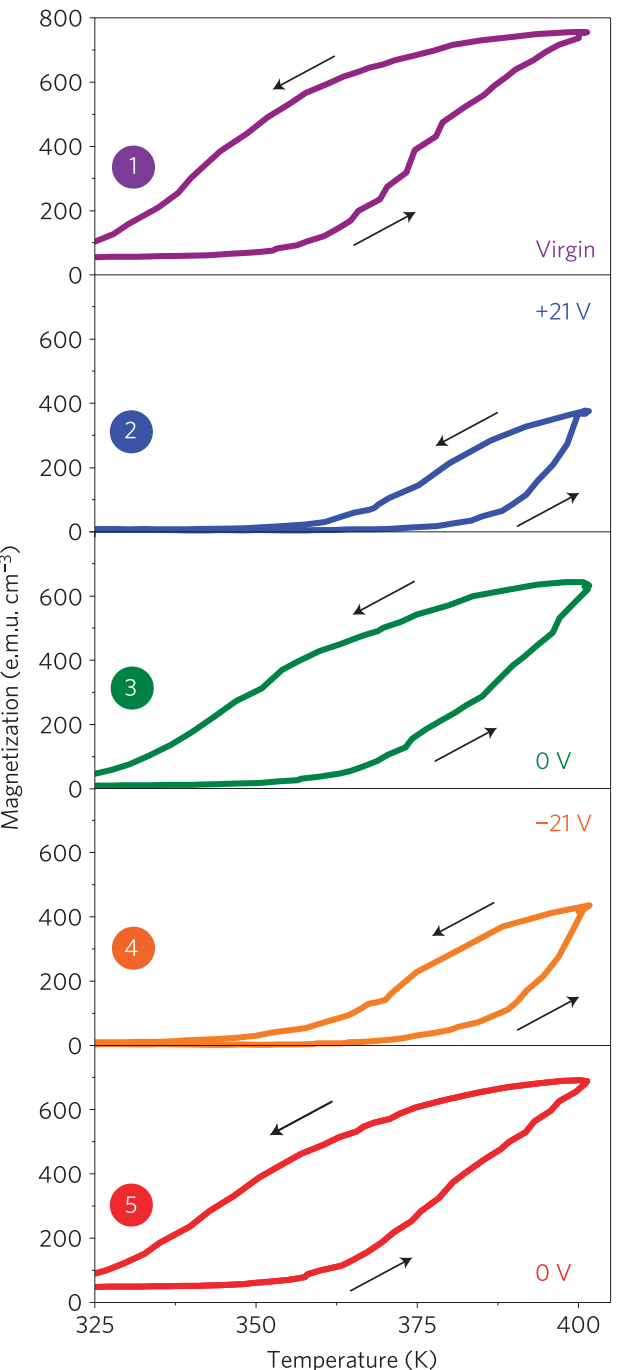

Figure 2 | Influence of an applied voltage on the temperature dependence of the magnetization in $\mathrm{FeRh}_{\mathbf{B}} \mathrm{BaTiO}_{\mathbf{3}}$. $\mathbf{a}$, $\mathrm{Polarization}$ versus voltage loop

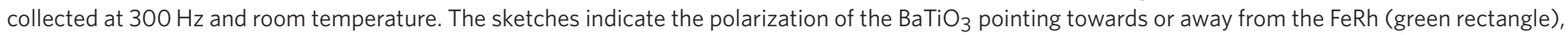

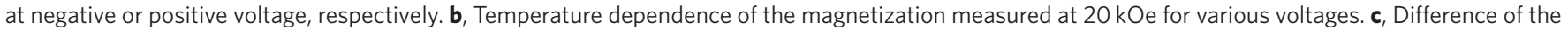
magnetization between selected curves from $\mathbf{b}$.

change, a reversible variation of $\Delta M \approx 70$ e.m.u. $\mathrm{cm}^{-3}$ is obtained. In this regime, the magnetization dependence is mainly symmetric, indicating that voltage-induced strain effects from $\mathrm{BaTiO}_{3}$ may dominate the response ${ }^{23}$.

$\mathrm{X}$-ray photoemission electron microscopy (XPEEM) offers a microscopic view of the same effect, as visible from the X-ray magnetic circular dichroism (XMCD) images measured at the $\mathrm{Fe}$ $\mathrm{L}_{3}$ edge of Fig. $3 \mathrm{~b}, \mathrm{c}$ for two different voltages at $385 \mathrm{~K}$. At $50 \mathrm{~V}$, no XMCD contrast is observed, in agreement with an AFM state of the FeRh. Switching the voltage off leads to the appearance of FM domains, as expected from a reduction of $T^{*}$ (ref. 24).

The role of voltage-induced strain effects is further confirmed by the data shown in Fig. 3d,e, which present X-ray diffraction $2 \theta-\omega$ scans near the $\mathrm{FeRh}(001)$, and $\mathrm{BaTiO}_{3}(002)$ and $(200) /(020)$ reflections, respectively, with and without an applied voltage, at $390 \mathrm{~K}$. The application of a voltage converts the $\mathrm{BaTiO}_{3}$ from a mixed state with a- and c-type domains (with a 34\%/66\% c-to-a domain population ratio) to a largely homogenous c-type configuration $(98 \% / 2 \%$ ratio), see Fig. 3e. We estimate that the applied voltage thus generates an in-plane compressive strain on the FeRh of $-0.47 \%$. Figure $3 \mathrm{~d}$ shows that the voltage produces a shift of the $\mathrm{FeRh}(001)$ reflection by $\sim 0.1^{\circ}$, which yields a voltage-induced elongation of the FeRh out-of-plane parameter by $0.52 \%$. Using a Poisson coefficient $v=0.31$, this corresponds to a compressive strain of $-0.47 \%$, which agrees with our estimate from the field-induced change in the domain population in the $\mathrm{BaTiO}_{3}$ (in the reversible regime).

The influence of the voltage on $c_{\mathrm{FeRh}}$ is also visible in the inset of Fig. 3a. A hysteretic variation of $c_{\mathrm{FeRh}}$ with voltage is obtained, consistent with the ferroelectric character of $\mathrm{BaTiO}_{3}$. The positions of the minima in this butterfly loop are in rather good agreement with those of the maxima from the voltage dependence of the magnetization (main panel of Fig. 3a), which further confirms that voltage-induced strain is driving the magnetization variation. We note that applying a voltage of $60 \mathrm{~V}$ at $440 \mathrm{~K}$ (well above the Curie point of $\mathrm{BaTiO}_{3}$ ) leaves $c_{\mathrm{FeRh}}$ unchanged, consistent with the paraelectric character of $\mathrm{BaTiO}_{3}$ at this temperature.

In the regime for which $M$ varies reversibly with voltage the magnetization values at the maximum positive and negative voltages are not equal. This may be the result of a slight asymmetry in the strain effect, but could also reflect the influence of another mechanism depending on the direction of the ferroelectric polarization, such as the field effect. In FeRh, the carrier density $n_{\mathrm{AFM}}$ in the AFM phase is unusually low, and by means of 
a

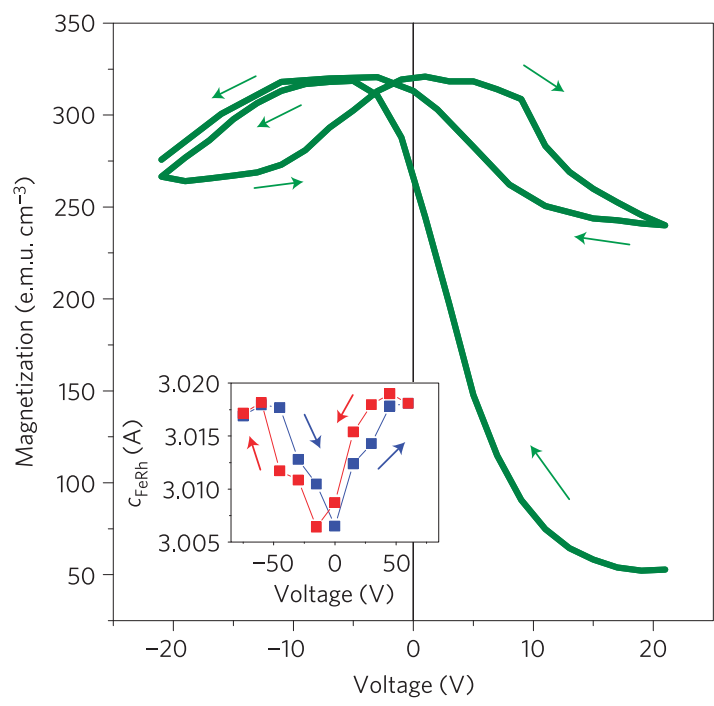

b
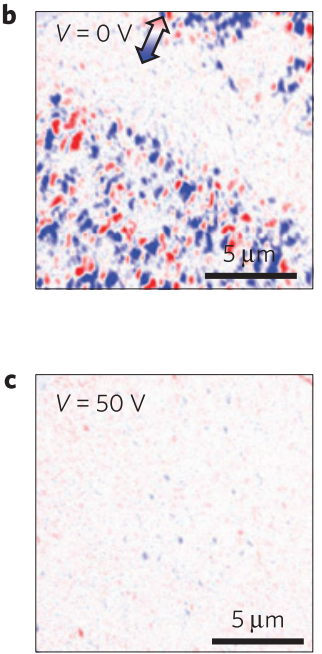

d
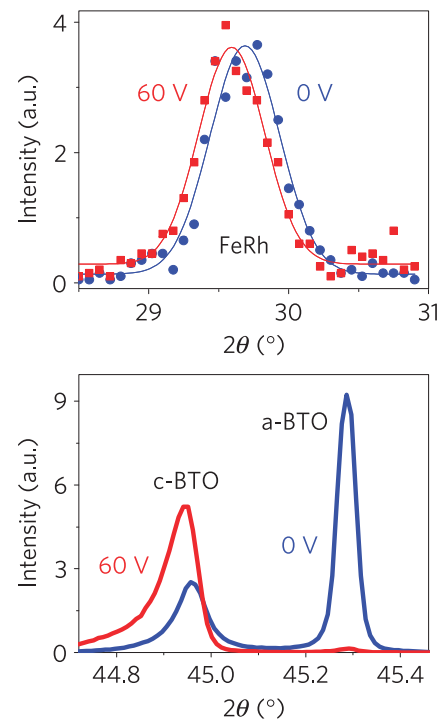

Figure 3 | Voltage dependence of the magnetization and the structural parameters. a, Variation of the magnetization with the applied voltage at $385 \mathrm{~K}$, after warming to this temperature in a voltage of $+21 \mathrm{~V}$. The inset shows the voltage dependence of the out-of-plane parameter of FeRh at $390 \mathrm{~K}$ b,c, XMCD-PEEM images at the Fe $\mathrm{L}_{3}$ edge at $0 \mathrm{~V}(\mathbf{b})$ and $50 \mathrm{~V}$ (c) and $385 \mathrm{~K}$. d,e, $2 \theta-\omega$ scans near the (001) reflection of FeRh (d) and the (002) and (200)/(020) reflections of $\mathrm{BaTiO}_{3}(\mathbf{e})$ at $390 \mathrm{~K}$, at $\mathrm{O}$ and $60 \mathrm{~V}$. In $\mathbf{d}$, the symbols correspond to the data and the lines to best fits using a pseudo-Voigt function.

Hall measurements at $300 \mathrm{~K}$ on similar films grown on $\mathrm{MgO}$ in the same conditions we measure $n_{\mathrm{AFM}}=3.5 \times 10^{21} \mathrm{~cm}^{-3}$. This is consistent with previous results extrapolated to samples with a high $\mathrm{Fe} / \mathrm{Rh}$ order ${ }^{12}$. Assuming a dielectric constant of 10 , we estimate a Thomas-Fermi screening length of $\lambda_{\mathrm{TF}} \approx 0.3 \mathrm{~nm}$. In a simple electrostatic model, charge accumulation/depletion effects and associated modifications of the physical properties induced by the $\mathrm{BaTiO}_{3}$ in the FeRh should thus occur over only a few unit cells. However, in magnetic materials, changes in the magnetic properties may be perceived over distances set by the exchange length $l_{\mathrm{ex}}$ (ref. 25), which is usually larger than $\lambda_{\mathrm{TF}}$ and can approach $10 \mathrm{~nm}$ (ref. 26). From this reasoning, electrostatic effects in $\mathrm{FeRh} / \mathrm{BaTiO}_{3}$ are thus expected to be small but non-zero and may contribute to the asymmetry in the voltage dependence of the magnetization shown in Fig. 3 a.

To gain a greater insight into the physical mechanisms responsible for the observed giant voltage-induced magnetization changes, we have performed first-principles calculations in the framework of density-functional theory. We first address the influence of strain on the properties of bulk FeRh. The red lines in Fig. 4a present the dependence of the internal energy as a function of the isotropic strain for the FM and AFM states (for both states the strain corresponds to the cell parameter change relative to that of the equilibrium AFM state). At zero strain, the AFM state is more stable (by $93 \mathrm{meV}$ ), as expected. The energy minimum for the FM state occurs at finite positive strain, which reflects the larger cell volume in the FM state compared to that of the AFM state.

The red curve in Fig. $4 \mathrm{~b}$ shows the difference $\Delta E=E_{\mathrm{FM}}-E_{\mathrm{AFM}}$ between the two energy curves (again, the AFM equilibrium cell parameter and internal energy have been taken as a reference for both magnetic states). We find a monotonic increase of $\Delta E$ as a function of compressive strain, which corresponds to a progressive stabilization of the AFM phase. This trend corresponds to the reported enhancement of $T^{*}$ with hydrostatic pressure ${ }^{9,11}$. For comparison, we reproduce in Fig. $4 \mathrm{~b}$ (square symbols, right axis) experimental values of $T^{*}$ as a function of the isotropic strain (estimated from the hydrostatic pressure and Young's modulus ${ }^{9}$ ). The agreement with the experimental data is excellent, which brings strong confidence in our theoretical approach. In addition,

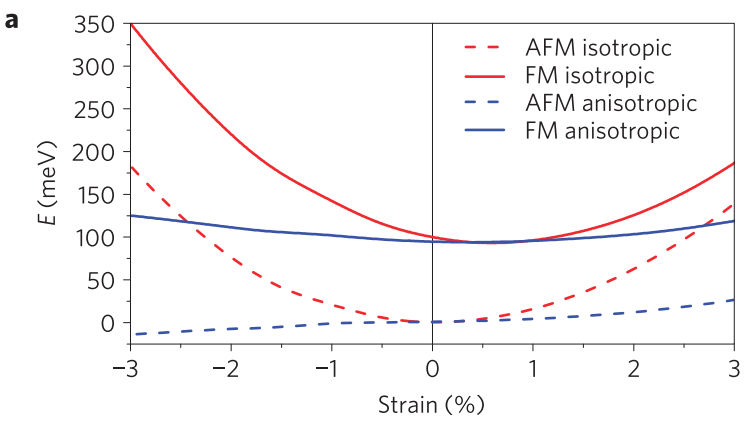

b

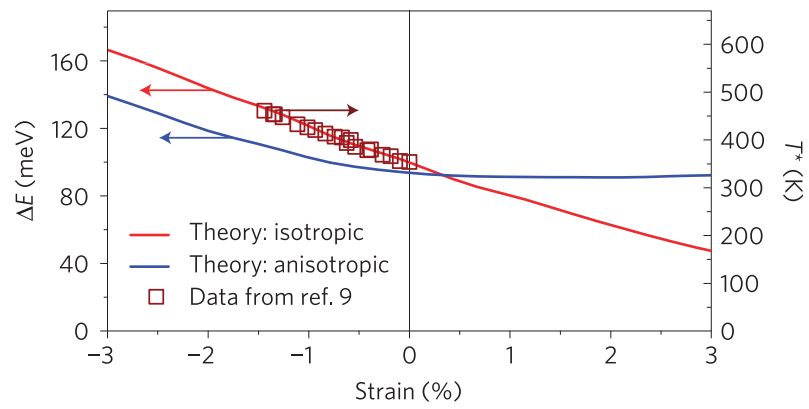

Figure 4 | Influence of strain on the stability of AFM and FM states.

a, Energy per FeRh unit formula versus the isotropic and anisotropic strain for AFM and FM bulk FeRh. b, Energy difference between the FM and AFM phases for the isotropic and anisotropic strain. The data for the transition $T^{*}$ temperature dependence versus isotropic strain are taken from ref. 9. The vertical scales for both axes are chosen to emphasize the correspondence between the theory and the data.

we have also considered both magnetic phases when subjected to an anisotropic strain which corresponds to the general strain conditions in epitaxial growth: in-plane parameters are constrained and the out-of-plane parameter is free to relax, resulting in a tetragonal distortion of the FeRh (blue lines, Fig. 4a,b). The strain dependence of energy is asymmetric and weak for tensile strain. For compressive strain, however, large $\Delta E$ variations are predicted. 
a

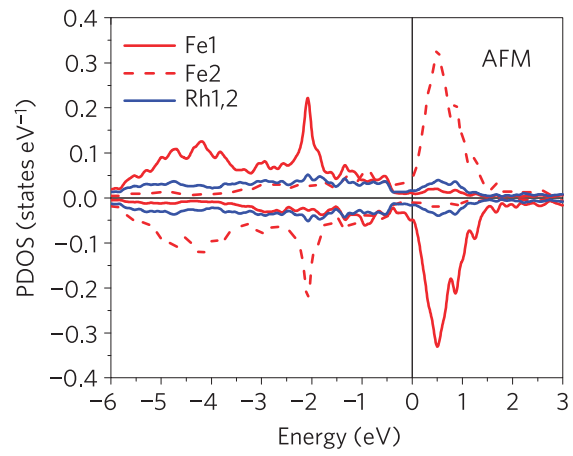

b

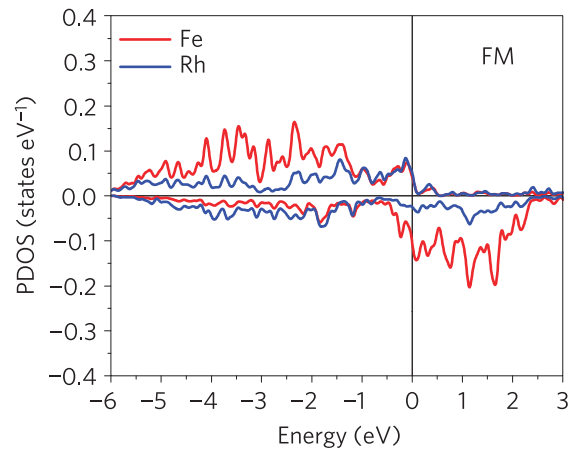

c

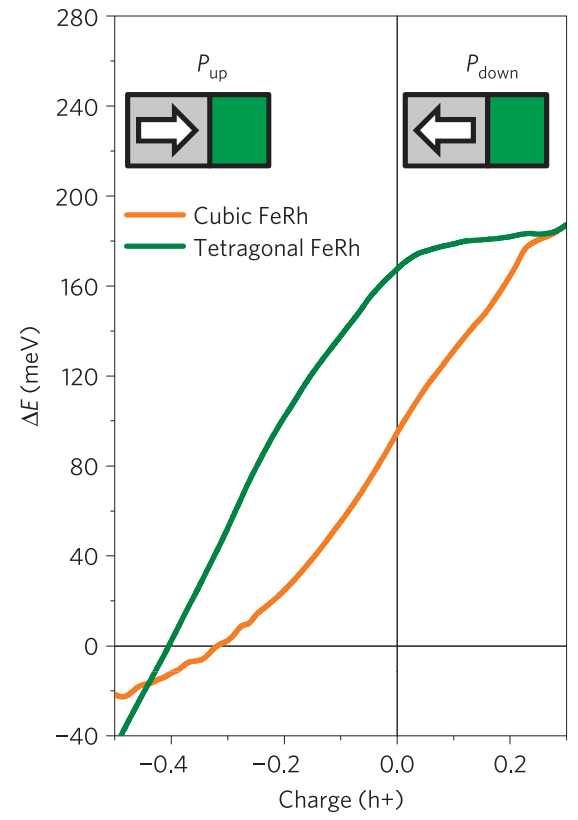

d

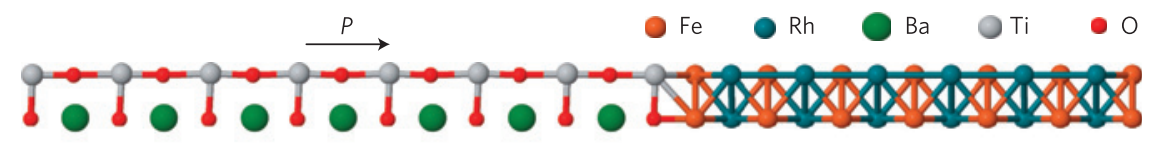

FM

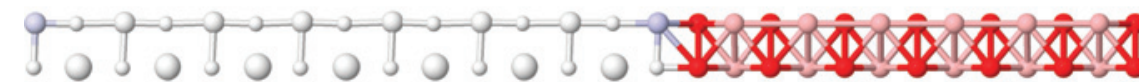

AFM

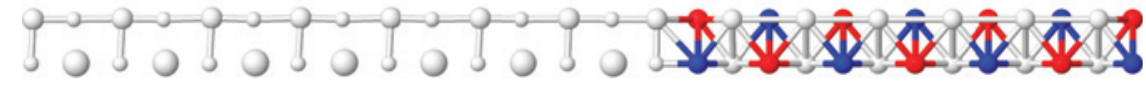

$\mathrm{AFM}^{\star}$

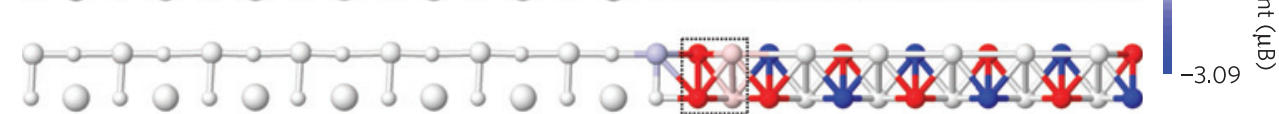

Figure $\mathbf{5}$ | Influence of charge injection on the magnetic order. a,b, Spin-resolved Fe 3d and Rh 4d PDOS for AFM (a) and FM (b) FeRh at the compressive strain expected for the interface. The Fermi level is set at zero energy. c, Energy difference per FeRh unit formula between the FM and AFM states as a function of the injected charge in hole $(\mathrm{h}+$ ) per interfacial unit cell. $\mathbf{d}$, Atomic structure of the FeRh/BaTiO 3 supercell (the direction of the ferroelectric polarization $P$ is denoted by the arrow) and optimized structures with different magnetic ordering. In the AFM* configuration the first FeRh unit cell at the $P_{\text {up }}$ interface is FM. The colour scale represents the local magnetic moment.

For a strain of $-0.47 \%$ and using the parallel trends of $\Delta E$ and $T^{*}$ inferred from the isotropic case, an increase of $T^{*}$ of about $20 \mathrm{~K}$ can be anticipated, in good agreement with the experimental results of Fig. $2 b$.

We now turn to the role of electrostatic effects. Figure 5a,b present spin-resolved partial densities of states (PDOS) projected on $\mathrm{Fe}$ and $\mathrm{Rh}$ sites. We find a much lower density of states at the Fermi level in the AFM state than in the FM state, consistent with previous calculations for bulk cubic FeRh (refs 13,27) and with Hall measurements ${ }^{12}$. As a consequence, a lower capability for charge accumulation and higher depolarization fields are expected at the $\mathrm{FeRh} / \mathrm{BaTiO}_{3}$ interface when FeRh is in the AFM state compared to when it is in the FM state. This effect is evident in Fig. 5c, which presents the energy difference between the FM and AFM states as a function of the injected charge (for both cubic and constrained FeRh) that would correspond practically to different values of the ferroelectric polarization in the $\mathrm{BaTiO}_{3}$. Whereas hole injection into $\mathrm{FeRh}$ does not significantly alter the relative stability between the two magnetic phases, electron injection progressively stabilizes the FM phase. One can thus expect that at the interface between $\mathrm{BaTiO}_{3}$ and $\mathrm{FeRh}$, the $P_{\text {down }}$ state (polarization away from the interface) has only a weak effect on the stability of the magnetic order but the $P_{\text {up }}$ configuration (polarization towards the interface) should promote the stabilization of ferromagnetism.

The relative stability of the two magnetic phases described here for bulk FeRh has also been investigated in the specific case of the interface with $\mathrm{BaTiO}_{3}$ at its equilibrium polarization. We consider the interface between Fe-terminated FeRh [001] and $\mathrm{TiO}_{2}$-terminated [001] $\mathrm{BaTiO}_{3}$, which corresponds to the most energetically favourable cutting plane. Metal atoms are located on top of oxygen atoms of the perovskite surface ${ }^{28}$, see Fig. $5 \mathrm{~d}$. The two interfaces of the supercell have the same composition but different relative orientations with respect to the ferroelectric polarization $P_{\text {up }}$ or $P_{\text {down }}$. As expected, the interface with the AFM slab is more stable than with the FM slab. However, to investigate more precisely the influence of the ferroelectric polarization on the interfacial magnetic state, we have also considered an $\mathrm{FeRh} / \mathrm{BaTiO}{ }_{3}$ supercell where the first FeRh unit cell at the $P_{\text {up }}$ interface was set to be FM (AFM* in Fig. 5d). We found that this structure is $100 \mathrm{meV}$ more stable than the structure for which the FeRh is entirely in 
the AFM state. A similar analysis at the $P_{\text {down }}$ interface gave a lower energy for the purely AFM configuration. This behaviour indicates a clear asymmetry between the $P_{\text {up }}$ and $P_{\text {down }}$ states and an easier transition to the FM state in the first case. This is consistent with our observation of a larger magnetization at $-21 \mathrm{~V}\left(P_{\text {up }}\right)$ than at $+21 \mathrm{~V}\left(P_{\text {down }}\right)$ in Fig. 3a.

In summary, we have found that in $\mathrm{FeRh} / \mathrm{BaTiO}_{3}$ a moderate electric field can produce a giant magnetization variation, arising from the electric-field-induced transformation of the FeRh from an AFM state to a FM state. The effect occurs just above room temperature and is mostly driven by voltage-induced strain effects from $\mathrm{BaTiO}_{3}$-but charge accumulation and depletion effects related to the ferroelectric polarization of $\mathrm{BaTiO}_{3}$ possibly contribute as well. In the future, it would be attractive to combine FeRh with piezoelectric elements with giant responses such as relaxors ${ }^{29}$, as our calculations suggest $T^{*}$ changes of $60 \mathrm{~K}$ for $1.5 \%$ strain. The effect could be further increased and tuned to a range of operating temperatures, including $300 \mathrm{~K}$, by using Pd-substituted FeRh (ref. 30), where we anticipate changes approaching $90 \mathrm{~K}$.

The possibility of toggling between AFM and FM states by means of an electric field and at very low power offers an attractive alternative to heat-assisted magnetic recording ${ }^{31}$. This technology uses a laser pulse to heat a magnetic bit above a certain temperature at which the magnetic field generated by the write-head can reliably switch the magnetization direction. A system of choice for such applications is FePt exchange biased to an FeRh-based alloy ${ }^{32}$, therefore our findings may spark the interest of industry in implementing and testing voltage-assisted alternatives using $\mathrm{THz}$ pulses ${ }^{33}$. On a broader perspective, our work emphasizes the relevance of hybrid perovskite/metal systems for low-power spintronic architectures.

\section{Methods}

Sample growth. FeRh films were grown by radiofrequency sputtering in a Plassys system at $630^{\circ} \mathrm{C}$ with a power of $45 \mathrm{~W}$ and in an argon pressure of $0.860 \mathrm{~Pa}$. The films were then annealed in situ at $730^{\circ} \mathrm{C}$ for $90 \mathrm{~min}$. During growth and annealing, the $\mathrm{BaTiO}_{3}$ crystals $\left(5 \times 5 \times 0.5 \mathrm{~mm}^{3}\right.$ in size and purchased from SurfaceNet $\mathrm{GmbH}$ ) were thus in the paraelectric cubic phase.

Structural and electrical measurements. X-ray diffraction was performed in a Panalytical Empyrean with $\mathrm{Cu} K \alpha 1$ radiation. For temperature- and voltage-dependent measurements we used a home-made two axis X-ray diffractometer in the Bragg-Brentano geometry with $\mathrm{Cu} \mathrm{K} \alpha 1$ radiation emitted by a $18 \mathrm{~kW}$ rotating anode (Rigaku). Polarization versus voltage loops were acquired with an Aixxact TF2000 Analyser.

Magnetometry. Magnetization measurements were carried out in a Quantum Design SQUID magnetometer with a maximum accessible temperature of $400 \mathrm{~K}$. The magneto-optical Kerr effect hysteresis loops were obtained using a $\lambda=408 \mathrm{~nm}$ laser diode in the longitudinal geometry at an incidence angle of about $45^{\circ}$. The magnetic field was applied in the incidence plane of the light and in the sample plane. The sample temperature was controlled, in air, using a home-made Joule-heated copper sample holder. The polarization state of the laser beam is modulated at $f=50 \mathrm{kHz}$ by a photo-elastic modulator such that the Kerr ellipticity and rotation can be measured at locked high frequencies $f$ and $2 f$, respectively, and normalized by the quasi-continuous incoming light intensity. It is highly sensitive to the magnetization within the skin depth region, typically $10-20 \mathrm{~nm}$

in most metals. In our experimental geometry, the Kerr ellipticity measured as a function of the applied field is mostly proportional to the net in-plane component of the magnetization.

\section{Photoemission electron microscopy with X-ray excitation (X-PEEM)}

High-resolution magnetic images were taken at the spin-resolved photo-emission electron microscope at the BESSY II storage ring in Berlin. This set-up is based on an Elmitec III instrument with an energy filter, permanently attached to an undulator beamline with full polarization control, in an energy range from 80 to $2,000 \mathrm{eV}$. The lateral resolution of the spin-resolved photo-emission electron microscope is about $25 \mathrm{~nm}$ for X-ray excitation. For magnetic imaging the photon energy was tuned to the $\mathrm{L}_{3}$ resonance of iron $(706.8 \mathrm{eV})$, exploiting the element-specific XMCD. Each of the XMCD images shown was calculated from a sequence of images taken with circular polarization $(90 \%$ of circular photon polarization) and alternating helicity. After normalization to a bright-field image, the sequence was drift-corrected, and frames recorded at the same photon energy and polarization have been averaged. The Fe magnetic contrast is shown as the difference of the two average images with opposite helicity, divided by their sum. The magnetic contrast represents the magnetization component pointing along the incidence direction of the X-ray beam. To collect the images in Fig. 3 the sample was first warmed from 300 to $390 \mathrm{~K}$ with the voltage applied, an image was then taken at $50 \mathrm{~V}$, and the voltage was switched off to take an another image at $0 \mathrm{~V}$.

First-principles calculations. Spin polarized density functional calculations under the local density approximation were performed as implemented in the AIMPRO code ${ }^{34}$. Relativistic pseudo-potentials were generated using the Hartwingster-Goedecker-Hutter scheme, resulting in basis sets of 50 independent Gaussian functions for iron and rhodium, 40 for titanium and oxygen and 20 for barium atoms. All atoms were optimized using a conjugate gradient scheme until the forces become less than $10^{-4} \mathrm{eV} \AA^{-1}$. Electronic structure convergence was assured by constraining the energy difference in the self-consistent cycle to be below $10^{-7}$ Hartree. We obtain for the body-centred cubic AFM FeRh a cell parameter of $2.922 \AA$ and for the FM phase a cell parameter of $2.935 \AA$. Magnetic moments on the Fe and $\mathrm{Rh}$ atoms are respectively 3.21 and $0.00 \mu \mathrm{B}$ in AFM FeRh and 3.29 and $0.94 \mu \mathrm{B}$ in FM FeRh, consistent with previous first-principles simulations ${ }^{27}$. For the tetragonal phase of $\mathrm{BaTiO}_{3}$ we obtain equilibrium lattice parameters of $a=3.945 \AA$ and $c / a=1.018$, in a good agreement with the experimental values ${ }^{17}$. To simulate the epitaxial growth of the metal onto the perovskite,

we fixed the FeRh in-plane cell parameter to the optimized value for $\mathrm{BaTiO}_{3}$ and left the out-of-plane supercell parameter free to relax simultaneously with the atomic positions.

Received 9 October 2013; accepted 18 December 2013; published online 26 January 2014

\section{References}

1. Chappert, C., Fert, A. \& Nguyen Van Dau, F. The emergence of spin electronics in data storage. Nature Mater. 6, 813-823 (2007).

2. Weiler, M. et al. Voltage controlled inversion of magnetic anisotropy in a ferromagnetic thin film at room temperature. New J. Phys. 11, 013021 (2009).

3. Lahtinen, T. H. E., Franke, K. J. A. \& van Dijken, S. Electric-field control of magnetic domain wall motion and local magnetization reversal. Sci. Rep. 2, 258 (2012)

4. Ghidini, M. et al. Non-volatile electrically-driven repeatable magnetization reversal with no applied magnetic field. Nature Commun. 4, 1421-1427 (2013).

5. Garcia, V. et al. Ferroelectric control of spin polarization. Science 327, 1106-1110 (2010)

6. Pantel, D., Goetze, S., Hesse, D. \& Alexe, M. Reversible electrical switching of spin polarization in multiferroic tunnel junctions. Nature Mater. 11, 289-293 (2012).

7. Chiba, D. et al. Electrical control of the ferromagnetic phase transition in cobalt at room temperature. Nature Mater. 10, 853-856 (2011).

8. Molegraaf, H. J. A. et al. Magnetoelectric effects in complex oxides with competing ground states. Adv. Mater. 21, 3470-3474 (2009).

9. Zakharov, A. I., Kadomtseva, A. M., Levitin, R. Z. \& Ponyatovskii, E. G. Magnetic and magnetoelastic properties of a metamagnetic iron-rhodium alloy. Sov. Phys. JETP 19, 1348-1353 (1964).

10. Stamm, C. et al. Antiferromagnetic-ferromagnetic phase transition in FeRh probed by x-ray magnetic circular dichroism. Phys. Rev. B 77, 184401 (2008).

11. Heeger, A. J. Pressure dependence of the FeRh first-order phase transition. J. Appl. Phys. 41, 4751-4752 (1970).

12. De Vries, M. A. et al. Hall-effect characterization of the metamagnetic transition in FeRh. New J. Phys. 15, 013008 (2013).

13. Gray, A. et al. Electronic structure changes across the metamagnetic transition in FeRh via hard X-ray photoemission. Phys. Rev. Lett. 108, 257208 (2012).

14. Vaz, C. A. F. Electric field control of magnetism in multiferroic heterostructures. J. Phys. Condens. Matter 24, 333201 (2012).

15. Naito, T., Suzuki, I., Itoh, M. \& Taniyama, T. Effect of spin polarized current on magnetic phase transition of ordered FeRh wires. J. Appl. Phys. 109, 07C911 (2011).

16. Cher, K. M., Zhou, T. J. \& Chen, J. S. Compositional effects on the structure and phase transition of epitaxial FeRh thin films. IEEE Trans. Magn. 47, 4033-4036 (2011).

17. Kay, H. F. \& Vousden, P. Symmetry changes in barium titanate at low temperatures and their relation to its ferroelectric properties. Phil. Mag. 40, 1019-1040 (1949)

18. Fan, R. et al. Ferromagnetism at the interfaces of antiferromagnetic FeRh epilayers. Phys. Rev. B 82, 184418 (2010).

19. Maat, S., Thiele, J-U. \& Fullerton, E. Temperature and field hysteresis of the antiferromagnetic-to-ferromagnetic phase transition in epitaxial FeRh films. Phys. Rev. B 72, 214432 (2005). 
20. Rado, G., Ferrari, J. \& Maisch, W. Magnetoelectric susceptibility and magnetic symmetry of magnetoelectrically annealed $\mathrm{TbPO}_{4}$. Phys. Rev. B 29, 4041-4048 (1984).

21. Eerenstein, W., Wiora, M., Prieto, J. L., Scott, J. F. \& Mathur, N. D. Giant sharp and persistent converse magnetoelectric effects in multiferroic epitaxial heterostructures. Nature Mater. 6, 348-351 (2007).

22. Zhang, S. et al. Electric-field control of nonvolatile magnetization in $\mathrm{Co}_{40} \mathrm{Fe}_{40} \mathrm{~B}_{20} / \mathrm{Pb}\left(\mathrm{Mg}_{1 / 3} \mathrm{Nb}_{2 / 3}\right)_{0.7} \mathrm{Ti}_{0.3} \mathrm{O}_{3}$ structure at room temperature. Phys. Rev. Lett. 108, 137203 (2012).

23. Hu, J-M., Nan, C-W. \& Chen, L-Q. Size-dependent electric voltage controlled magnetic anisotropy in multiferroic heterostructures: Interface-charge and strain comediated magnetoelectric coupling. Phys. Rev. B 83, 134408 (2011).

24. Baldasseroni, C. et al. Temperature-driven nucleation of ferromagnetic domains in FeRh thin films. Appl. Phys. Lett. 100, 262401 (2012).

25. Ovchinnikov, I. \& Wang, K. Theory of electric-field-controlled surface ferromagnetic transition in metals. Phys. Rev. B 79, 020402(R) (2009).

26. Abo, G. S. et al. Definition of magnetic exchange length. IEEE Trans. Magn. 49, 4937-4939 (2013)

27. Sandratskii, L. M. \& Mavropoulos, P. Magnetic excitations and femtomagnetism of FeRh: A first-principles study. Phys. Rev. B 83, 174408 (2011).

28. Meyerheim, H. et al. Structural secrets of multiferroic interfaces. Phys. Rev. Lett. 106, 2-5 (2011)

29. Park, S-E. \& Shrout, T. R. Ultrahigh strain and piezoelectric behavior in relaxor based ferroelectric single crystals. J. Appl. Phys. 82, 1804-1811 (1997).

30. Wayne, R. Pressure dependence of the magnetic transitions in Fe-Rh alloys. Phys. Rev. 170, 523-527 (1968).

31. Challener, W. A. et al. Heat-assisted magnetic recording by a near-field transducer with efficient optical energy transfer. Nature Photon. 3, 220-224 (2009).

32. Thiele, J-U., Maat, S. \& Fullerton, E. E. FeRh/FePt exchange spring films for thermally assisted magnetic recording media. Appl. Phys. Lett. 82, 2859-2861 (2003).
33. Hirori, H., Doi, A., Blanchard, F. \& Tanaka, K. Single-cycle terahertz pulses with amplitudes exceeding $1 \mathrm{MV} / \mathrm{cm}$ generated by optical rectification in $\mathrm{LiNbO}_{3}$. Appl. Phys. Lett. 98, 091106 (2011).

34. Rayson, M. J. \& Briddon, P. R. Rapid iterative method for electronic-structure eigenproblems using localised basis functions. Comput. Phys. Commun. 178, 128-134 (2008).

\section{Acknowledgements}

We are very grateful to A. Gloter and S. Van Dijken for fruitful discussions and R. Mattana, C. Carrétéro, E. Lesne and R. Weil for technical assistance with SQUID measurements, sample growth and high-temperature MOKE. This work received financial support from the French Agence Nationale de la Recherche through project NOMILOPS (ANR-11-BS10-0016) and the European Research Council Advanced Grant FEMMES (contract no. 267579). R.O.C. acknowledges financial support by Thales through a CIFRE PhD grant.

\section{Author contributions}

M.B. and A.B. initiated the study. A.B. and R.O.C. conceived the experiments. R.O.C. prepared the samples and performed RHEED with the assistance of E.J. L.C.P., I.C.I., B.D., N.G., R.O.C. and M.B. carried out the X-ray diffraction experiments. V.G., S.F. and R.O.C. measured the ferroelectric response of the samples. R.O.C. characterized the samples by SQUID magnetometry, A.M. by MOKE and L.C.P., A.A.Ü., S.V. and F.K. by X-PEEM. V.I. and A.Z. performed the first-principles calculations using the code developed by P.R.B. M.B. wrote the manuscript with inputs from V.I. and A.Z. All authors contributed to the manuscript and the interpretation of the data.

\section{Additional information}

Reprints and permissions information is available online at www.nature.com/reprints. Correspondence and requests for materials should be addressed to M.B.

\section{Competing financial interests}

The authors declare no competing financial interests. 